\title{
Information Technologies for Distributed Machine Drives: an Overview
}

\author{
Filippo Savi, Giampaolo Buticchi \\ Chris Gerada, Patrick Wheeler \\ Department of electrical engineering \\ University of Nottingham \\ Ningbo, China
}

\author{
Davide Barater \\ Dipartimento di Ingegneria "Enzo Ferrari" \\ Università degli studi di Modena e Reggio Emilia \\ Modena, Italy
}

\begin{abstract}
The exponential growth experienced by the semiconductor manufacturing field has led to a large proliferation of devices with large amounts computational power, enabling countless technologies and revolutionizing many fields. Control systems and machine drives are certainly among them. Much research is being carried out to develop multi-phase and fully segmented machines, with their inherent fault tolerance. To take full advantage of the redundancy and load sharing capabilities of the machine structure, with multiple winding sets, a suitable distributed control method must be used. A high performance network between the drives is thus required.

This paper will present an overview of the available communication protocols that are used in the field and evaluate how suitable are they to this new class of very demanding real time tasks.
\end{abstract}

\section{INTRODUCTION}

In many cases the use of a distributed machine drives can be highly advantageous, with potentially better performances, lower costs and higher reliability. A renewed interest in these systems has been sparked in the aerospace sector due to More electric aircraft (MEA)initiatives [5]. The end goal of MEA is the complete electrification of most aircraft subsystems improving overall efficiency, for better fuel efficiency and reduced emissions. On currently flying aircraft a multitude of power transmission methods are used for different tasks: mechanical power is used to drive pumps and other ancillary equipment in the main engines, pneumatic power in the form of hot compressed air is used for anti-icing and environmental control systems lastly hydraulic power is used throughout the aircraft for: primary and secondary flight surfaces, landing gear movement and several other ancillary equipment. The move to a fully electric power distribution network, presents several advantages among which: weight savings from the removal of hydraulic piping and reservoirs, less and easier maintenance due to the reduction of moving parts and seal, better main engine efficiency with a bleed-less design where no power is extracted from the jet engine between compressor and combustor sections.

In order to achieve the previously outlined goals the need has arisen for high density and fault tolerant electric machines. While to increase power density, along with high performance magnetic materials, operating speed of machines is increased, the most promising method for achieving partial fault tolerance is the use of multi-phase or fully segmented machines. Here the stator winding is split into more than three phases, or even replicated in multiple unconnected sub-units that are radially or axially separated. In case of a fault the damaged winding can thus be isolated allowing the remaining ones to keep on working, eventually at a reduced speed. A key component in this system is a distributed drive system that can fully control the machine, as an uneven stator load can lead to premature thermal degradation of the insulation system, with a net decrease of overall reliability. 
One of the main requirement for a high performance distributed machine drive control system to work is the availability of a low latency communication channel upon which the various drives can exchange the required information. This paper presents an overview of the communication protocols commonly used for machine drives control analyzing their performances when presented with the high control loop frequencies required in these applications. In section III the main control strategies for distributed drives are reviewed, in section III the figures of merit used in the comparison are presented and in section $[\mathrm{IV}$ the results are presented.

In this digest, only the channel capacity analysis is presented, more results will be available in the full paper

\section{Distributed MACHINE DRIVE SYSTEMS}

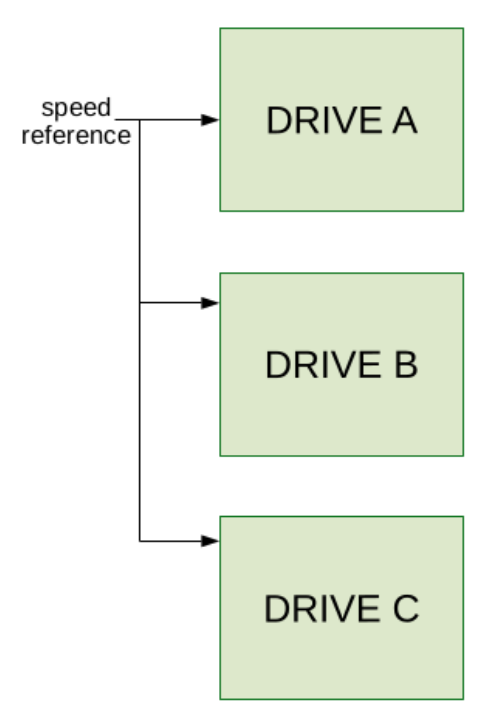

(a)

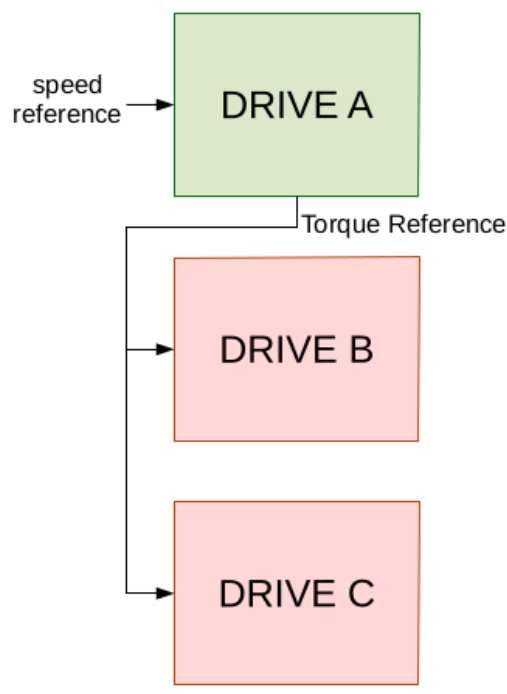

(b)

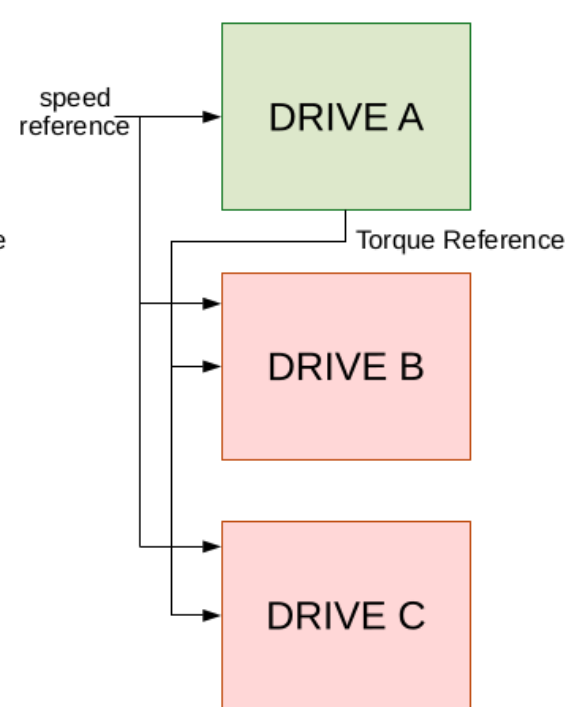

(c)

Figure 1: Distributed drives control topologies (a) Common Speed Reference (b) Torque Follower (c) Speed Trim Follower

In Figure 1, are shown the three control schemes that can be employed in this type of systems, as also shown in [1]: Common speed reference, Torque follower and speed-trim follower. In the first strategy the drives are completely unconnected to each other and a speed droop coefficient is added to achieve some load sharing. While very simple the performance of this control system are sub-optimal, since load sharing is not precise and speed regulation is poor, especially when a lot of machines/segments are involved. The second strategy, the Torque Follower, employs a master-slave arrangement, where the master regulates the speed and directly controls the slave drives (shown in green in Figure 1) with a torque set point. A similar control method is the Speed-Trim Follower where both master and slaves work in speed regulation mode, whith the latter ones having a set point that is function of both the input speed and a torque setting from the master. In both the latter cases interconnections between drives is necessary, and thus a reliable, fault tolerant and low latency communication protocol is required. 


\section{COMMUNICATIONS PERFORMANCE METRICS FOR DISTRIBUTED DRIVES}

A key enabling technology for distributed electrical drive systems, is a high performance communication network between the nodes. The importance of this components while not always acknowledged is crucial, both for the correct working of the overall strategy and to the control performances. In order to compare several communication protocols, a set of performance metrics is needed. A first, extremely important figure of merit, especially for control systems, is end-to-end delay, defined as the time needed for a network transaction to reach full completion. This time is however not an homogeneous quantity [3], as it incorporates the contributes shown in equation 1 . $\tau_{P R O C}$ is the delay due to all the processing en route, $\tau_{T R A N S}$ is the transmission time dependent on the channel's bit rate, $\tau_{P R O P}$ is the physical propagation delay and $n$ is the number of routing hops from source to destination. Of this components the dominant one is transmission time.

$$
\tau_{E E}=n * \tau_{P R O C}+n * \tau_{T R A N S}+n * \tau_{P R O P}
$$

Another very important metric is channel capacity, that in this setting we can define as the number of nodes that can be supported by the communication protocol. This depends both on intrinsic characteristics of the physical channel, like bit rate and operating conditions such as the control loop frequency and the amount of data to be exchanged at each step. For this study three different control loop frequencies, have been considered:500 Hz, $1 \mathrm{kHz}$ and $5 \mathrm{kHz}$. With the first one being suitable for constant of slow varying loads, while the highest one is needed for high dynamics applications. The data that need to be exchanged between the drives at each cycle is listed below, with each field's length being 16 bits.

\section{- Common Speed reference}

- Position sensor reading

\section{- Torque Follower}

- Position sensor reading

- Speed/Torque reference

\section{- Speed Trim Follower}

- Position sensor reading

- Torque reference

- speed reference

The last considered performance figure is protocol efficiency [6], defined in equation 2, as the ratio of payload data length to overall packet length. This metric has a large impact on the overall system design; when considering channels with limited bit rates, a higher efficiency translates directly to a larger number of supportable nodes or lower transmission delays. Even when no limits are in place,a high efficiency can lead to lower system cost, as more expensive cabling and transceivers are needed for increasing channel bandwidth.

$$
\eta=\frac{l_{\text {payload }}}{l_{\text {packet }}}
$$




\begin{tabular}{|c|c|}
\hline Protocol & Bit rates \\
\hline Modbus RTU & $115.2 \mathrm{~kb} / \mathrm{s}$ \\
\hline CAN 2.0A & $10 \mathrm{Mb} / \mathrm{s}$ \\
\hline CAN 2.0B & $10 \mathrm{Mb} / \mathrm{s}$ \\
\hline Profinet & $100 \mathrm{Mb} / \mathrm{s}$ \\
\hline
\end{tabular}

Table 1: Maximum bit rates of the examined protocols

\section{STATE OF THE ART OVERVIEW}

\section{i. protocol choice}

A large number of communication methods are used to interface machine drives with the outside world, both for simple command tasks and for distributed control. This analysis will consider the most general and widely used among them, ignoring the ones that have been superseded or while still in use, are limited to only a narrow niche of market. The desire of manufacturer to retain full control over the full product has lead to a proliferation of similar communication protocols that are based on few common lower layers standards, like RS-485/232, CAN, etc. This situation is especially prominent when it comes to Ethernet, as a large number of competing standards with largely similar performances and functionality are pushed by different PLC manufacturers. In this study only one of them has been considered. The protocols examined in the following sections will be: Modbus RTU [4], as it is still in widespread usage, especially in the industrial automation field. The second protocol chosen is CAN bus [2], in both it's normal (CAN 2.0A) and extended (CAN 2.0B) addressing variants, as it is used, not only in the automotive sector, but also in maritime, rail and aerospace fields, respectively NMEA2000 and ARINC 825. The last communication protocols taken in consideration is profinet IRT, this protocol is relatively modern and is one of the many industrial automation protocols based on the Ethernet family of standards.

\section{ii. Channel capacity}

When comparing results from the channel capacity analysis, several trends appear. Modbus RTU is limited by the low speed and efficiency of the RS-485 protocol is built upon, in this case the only usable control method is the common speed reference. The other protocols are all capable of supporting every examined control strategy, with a maximum system size that depends on the protocol performances. It is also worth noting that for this peculiar class of applications, the CAN 2.0B protocol is worse with respect to the A variant; the longer message ID field introduces additional latency but does not improve the number of channels that can be supported since the low maximum bit-rate is a limiting factor. The Ethernet based Profinet, despite its larger packet size and higher complexity is clearly the most capable protocol, as it can support very large systems with many tens of drives even at the higher control loop frequencies.

\footnotetext{
${ }^{1}$ This bit rate is while non standard, in widespread use, higher bit rates while possible are not commonly supported
} 


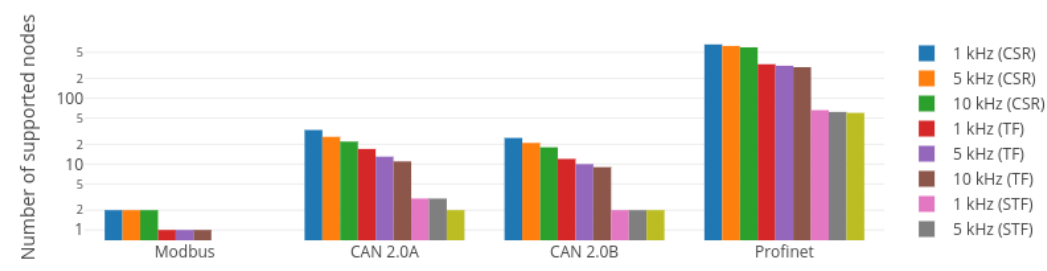

Figure 2: Number of supported channels at various control loop frequencies

\section{Conclusions}

In this paper we reviewed the principal communication protocols that are used for machine drives and evaluated their performances in distributed drive control system. It was clear that the Ethernet based protocols, of which Profinet is an example can provide high throughput while maintaining lower latencies than other protocols, a key advantage for real time control applications.

\section{REFERENCES}

[1] Allen Bradley. Load Sharing for the 1336 PLUS II AC Drive, 2000.

[2] Road vehicles - Controller area network (CAN) - Part 2: High-speed medium access unit. Standard, International Organization for Standardization, Geneva, CH, December 2006.

[3] James F. author Kurose. Computer networking : a top-down approach / James F. Kurose, University of Massachusetts, Amherst, Keith W. Ross, NYU and NYU Shanghai. Seventh edition. edition, 2017.

[4] The modbus organization. MODBUS over Serial Line Specification and Implementation Guide. Technical report, 2006.

[5] J. A. Rosero, J. A. Ortega, E. Aldabas, and L. Romeral. Moving towards a more electric aircraft. IEEE Aerospace and Electronic Systems Magazine, 22(3):3-9, 2007.

[6] P. Truchly and M. Beragg. Transmission efficiency of current communications systems. In 2007 14th International Workshop on Systems, Signals and Image Processing and 6th EURASIP Conference focused on Speech and Image Processing, Multimedia Communications and Services, pages 491-494, June 2007. 